\title{
Efficacy of epidermal growth factor receptor-tyrosine kinase inhibitors for patient with leptomeningeal metastasis of epidermal growth factor receptor mutant non-small cell lung cancer
}

\author{
Jong Sik Lee, Kyung Ann Lee, Kang Hoon Lee, Sun Young Moon, In Ae Kim, Sung Jin Jeon, \\ Jae Ki Min, Hee Joung Kim, Kye Young Lee \\ Department of Internal Medicine, Konkuk University School of Medicine, Seoul, Korea
}

We report on a 64-year-old man with leptomeningeal metastasis (LM) from an epidermal growth factor receptor (EGFR)-mutated adenocarcinoma of the lung. He was treated with paclitaxel, cisplatin. After completion of chemotherapy, he complained of headache, nausea, and vomiting. EGFR-mutated tumor cells were identified from the cerebrospinal fluid (CSF). Second-line therapy with gefitinib, methotrexate was started. After receiving gefitinib for 4 weeks, he had no more headaches or vomiting. Eleven months after initiation of gefitinib, he developed headache and nausea. Chest computed tomography showed aggravation of bone metastasis. Third-line therapy was started with gemcitabine and carboplatin. Two weeks later, he experienced disorientation. After a fourth relapse within the central nervous system, the therapy was switched to erlotinib and significant improvement of LM was achieved. This case shows that LM can be diagnosed by detecting EGFR mutation in CSF and EGFR tyrosine kinase inhibitors are effective for LM from EGFR mutant non-small cell lung cancer.

Keywords: Epidermal growth factor receptor; Cerebrospinal fluid; Gefitinib; Erlotinib

\section{INTRODUCTION}

Leptomeningeal metastasis (LM) is a serious complication of cancer with very poor prognosis. The frequency of $\mathrm{LM}$ has been reported to be $10 \%$ to $25 \%$ of lung cancer patients [1], and it is more common in small cell lung cancer than in non-small cell lung cancer. Several mechanisms of leptomeningeal spreading have been proposed: (1) hematogenous spread via arachnoid vessels, (2) direct extension from parenchymal or parameningeal tumors, and (3) iatrogenic dissemination during surgery for parenchymal metastasis. The diagnostic evaluation of LM should be performed by gadoliniumenhanced brain magnetic resonance imaging (MRI) and cere-

Received: December 23, 2014, Revised: April 14, 2015 Accepted: April 21, 2015

Corresponding Author: Kye Young Lee, Department of Internal Medicine, Konkuk University School of Medicine, 120-1, Neungdong-ro, Gwangjin-gu, Seoul 05030, Korea Tel: +82-2-2030-7521, Fax: +82-2-2030-7748

E-mail: kyleemd@kuh.ac.kr brospinal fluid (CSF) studies. Intrathecal chemotherapy and radiotherapy are the standard therapies, and the treatment goal is generally only the palliation of symptoms.

Sequence alterations in the epidermal growth factor receptor (EGFR) gene have frequently been identified in non-small cell lung cancer, primarily in patients who are responsive to gefitinib (Iressa), an EGFR tyrosine kinase inhibitor (TKI) [2,3]. There are few case reports showing EGFR mutation in metastatic brain lesions, which are rare in CSF cytology $[4,5]$.

This report describes a patient with EGFR mutant non-small cell lung cancer who showed significant improvement of LM to erlotinib after failure of gefitinib.

\section{CASE}

A 64-year-old man with a 13-pack-per-year smoking history was diagnosed with stage IV adenocarcinoma of the lung with multiple bone metastases. He presented with blood-tinged sputum and right posterior chest wall pain. Chest computed tomography (CT) confirmed a $2.0 \times 2.3 \mathrm{~cm}$ central mass with 
satellite nodules in the right lower lobe and enlarged mediastinal and supraclavicular lymph nodes. The patient underwent a bronchoscopy and a biopsy of the endobronchial mass in the right lower lobe. The tumor specimen revealed adenocarcinoma with an in-frame deletion in exon 19 (2239 T>C). The patient received first-line therapy with six cycles of paclitaxel and cisplatin, which resulted in a partial response. However, 3 months after completion of cytotoxic chemotherapy, the patient complained of headache, nausea, vomiting and fever. A brain MRI showed diffuse LM and right occipital bone metastasis with diffuse marrow infiltration (Fig. 1).

The patient had no respiratory symptoms, and the chest CT scan showed no evidence of local recurrence. A lumbar puncture was performed, and LM was confirmed by CSF cytologic examination (Fig. 2). We identified the combination of a point mutation and a deletion in exon 19 (del 2240-2251, 2239 T>C; L747-T751insP; heterozygote) in tumor cells from the
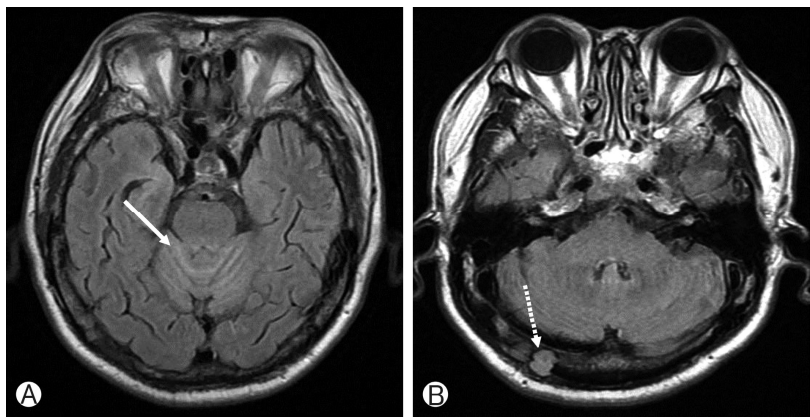

Fig. 1. T2 FLAIR contrast enhancement brain MRI upon diagnosis of leptomeningeal metastasis. (A) The image shows diffuse metastatic enhancement of both cerebellar fissures (solid white arrow), (B) a nodular mass on the right occipital bone (dotted white arrow). FLAIR, fluid-attenuated inversion recovery; MRI, magnetic resonance imaging.
CSF. The patient was administered gefitinib $(250 \mathrm{mg}$ per daily) and intrathecal chemotherapy with methotrexate through an Ommaya reservoir (15 mg twice per week). After 4 weeks of gefitinib administration, he had no more headaches or episodes of vomiting. A brain MRI showed significant improvement of LM (Fig. 3), and there were no malignant cells in the CSF. Eleven months after initiation of gefitinib treatment, the patient developed headaches and nausea. A brain MRI follow-up scan showed mixed LM responses, and a chest CT scan showed aggravation of the right 7 rib destruction. However, there were no malignant cells in the CSF. Gefitinib was discontinued, and third-line therapy was started with gemcitabine and carboplatin. Two weeks later, the patient experienced an episode of disorientation. The CSF cytology was reevaluated and demonstrated malignant tumor cells. The brain MRI also indicated disease progression with newly developed hydrocephalus. Erlotinib was started, and a ventriculoperito-
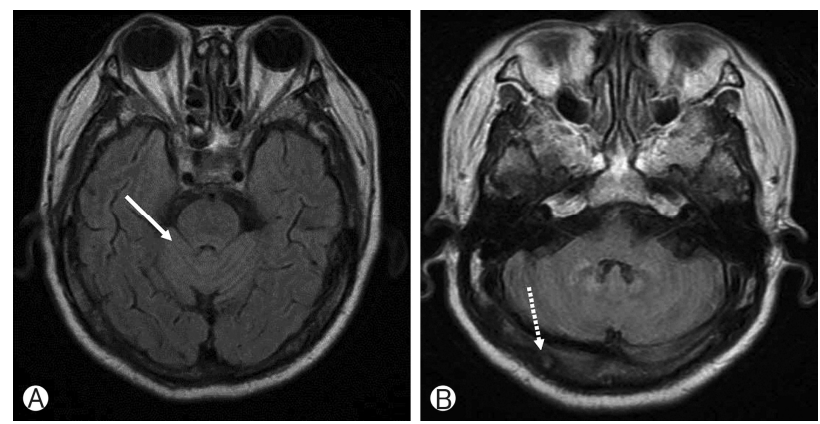

Fig. 3. Responses to gefitinib of leptomeningeal metastasis and bone metastasis on the right occipital bone (T2 FLAIR contrast enhancement). (A) No residual metastatic enhancement was observed (solid white arrow), and (B) a decreased nodular mass was found (dotted white arrow). FLAIR, fluid-attenuated inversion recovery.

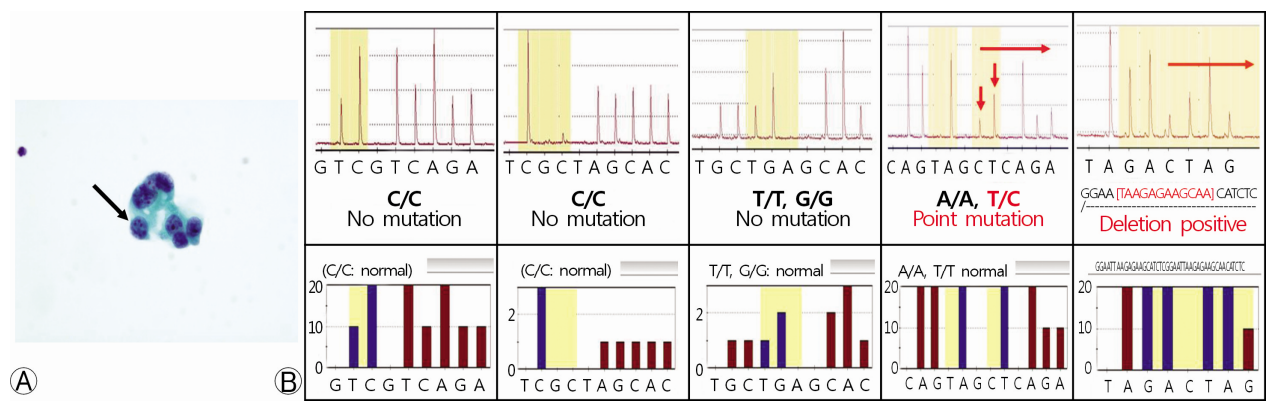

Fig. 2. (A) Cytology specimen for cerebrospinal fluid shows metastatic adenocarcinoma cells (black arrow) (Papanicolaou stain). (B) In DNA extracted from CSF, EGFR mutation analysis using pyrosequencing showed a point mutation and deletion in exon 19. CSF, cerebrospinal fluid; EGFR, epidermal growth factor receptor. 
neal shunt operation was performed. The patient exhibited neither neurological symptoms nor adverse effects of erlotinib at the 8-week follow-up examination.

\section{DISCUSSION}

The prognosis for patients with $\mathrm{LM}$ is poor, with a median survival of 4-6 weeks if left untreated and 2-3 months with aggressive therapy [6]. Moreover, the treatment of LM is not curative, and radiation therapy and intrathecal chemotherapy can offer palliation by arresting neurological progression in clinically stable patients [7]. However, the median overall survival is usually less than 6 months. Systemic chemotherapy agents including thiotepa, methotrexate and cytarabine can penetrate the blood-brain barrier (BBB). Nevertheless, intrathecal chemotherapy has been the mainstay of treatment in LM [1]. Some recent studies have shown that EGFR-TKIs may be effective in patients with brain metastasis [8,9].

EGFR gene mutations, particularly exon 19 deletions and exon 21 (L858R or L861Q) or 18 (G719X) mutations, are the best predictors of the patients' responses to EGFR-TKIs. Pretreatment EGFR mutation testing in routine practice is essential to ensure that all patients with EGFR-sensitizing mutations benefit from EGFR-TKIs.

LM is known to have high specificity for detection of malignant cells in the CSF through cytology. However, it has a low sensitivity (50\% to 60\%) in patients with autopsy-proven LM. Because LM contains very few morphologically recognizable malignant cells, making a definitive diagnosis of LM by CSF cytology is difficult. Pyrosequencing technology offers an opportunity for tumor diagnosis based on detection of tumor-derived DNA present in the CSF, even if malignant cells cannot be detected by cytological investigation of the CSF [10-14]. Adequate cytology specimens as well as biopsy specimens are sufficient for molecular analysis [15], and will be very important for patients to receive personalized targeted therapy based on their molecular profiles.

Gefitinib is an orally active and selective EGFR-TKI that blocks the signal transduction pathways implicated in promoting cancer growth, and thus, this treatment leads to decreased cellular proliferation, angiogenesis, tumor invasion and metastasis and increased apoptosis.

Several recent reports have described the effective treatment of LM with EGFR-TKIs. Jackman et al. described a case of
LM in a patient with non-small cell lung cancer with activating EGFR mutation treated with a high dose of gefitinib. These researchers suggested a correlation between a higher concentration of gefitinib in the CSF and the subsequent clinical and radiographical response of LM [4]. Choong et al. [5] reported on a patient with erlotinib-refractory lung adenocarcinoma who developed LM and responded well to gefitinib.

This case showed significant response to erlotinib in EGFR mutant non-small cell lung cancer with LM, even after developing resistance to gefitinib.

This case highlights some conclusions regarding targeted therapy with TKIs for LM of non-small cell lung cancer. First, the usual dose of gefitinib ( $250 \mathrm{mg}$ per day) would be effective for treatment of LM with an activating EGFR mutation, as confirmed by a CSF analysis. Our patient had no cytochrome P4503A4 inhibitors that may increase the level of gefitinib. Jackman et al. [4] reported some adverse effects of gefitinib, including somnolence and increased hepatic transaminase with the dose of $750 \mathrm{mg}$ per day. However, our patient showed a dramatic response to gefitinib within 3 months, and the disease was effectively controlled over a period of 11 months with no severe adverse effects. Second, gefitinib could penetrate the BBB without obvious disruption of the BBB. Kim et al. [8] described a patient with LM in non-small cell lung cancer who showed good response to gefitinib after undergoing laminectomy for resection of spinal metastatic tumors. In contrast, our patient presented no evidence of BBB disruption, such as spinal or brain surgery. Therefore, we can hypothesize that gefitinib may be able to penetrate the BBB under LM conditions, although the entire dose of gefitinib cannot penetrate the common BBB.

Further investigations are required to elucidate the mechanisms through which gefitinib can penetrate the BBB and through which gefitinib results in resistance to EGFR-TKIs in LM.

\section{REFERENCES}

1. O'Meara WP, Borkar SA, Stambuk HE, Lymberis SC. Leptomeningeal metastasis. Curr Probl Cancer 2007;31:367-424.

2. Lynch TJ, Bell DW, Sordella R, Gurubhagavatula S, Okimoto $\mathrm{RA}$, Brannigan BW, et al. Activating mutations in the epidermal growth factor receptor underlying responsiveness of non-small-cell lung cancer to gefitinib. N Engl J Med 2004; 350:2129-39. 
3. Tokumo M, Toyooka S, Kiura K, Shigematsu H, Tomii K, Aoe $\mathrm{M}$, et al. The relationship between epidermal growth factor receptor mutations and clinicopathologic features in non-small cell lung cancers. Clin Cancer Res 2005;11:1167-73.

4. Jackman DM, Holmes AJ, Lindeman N, Wen PY, Kesari S, Borras AM, et al. Response and resistance in a non-small-cell lung cancer patient with an epidermal growth factor receptor mutation and leptomeningeal metastases treated with highdose gefitinib. J Clin Oncol 2006;24:4517-20.

5. Choong NW, Dietrich S, Seiwert TY, Tretiakova MS, Nallasura V, Davies GC, et al. Gefitinib response of erlotinib-refractory lung cancer involving meninges--role of EGFR mutation. Nat Clin Pract Oncol 2006;3:50-7.

6. Wasserstrom WR, Glass JP, Posner JB. Diagnosis and treatment of leptomeningeal metastases from solid tumors: experience with 90 patients. Cancer 1982;49:759-72.

7. Glantz MJ, Jaeckle KA, Chamberlain MC, Phuphanich S, Recht L, Swinnen LJ, et al. A randomized controlled trial comparing intrathecal sustained-release cytarabine (DepoCyt) to intrathecal methotrexate in patients with neoplastic meningitis from solid tumors. Clin Cancer Res 1999;5:3394-402.

8. Kim MK, Lee KH, Lee JK, Choi JH, Hyun MS. Gefitinib is also active for carcinomatous meningitis in NSCLC. Lung Cancer 2005;50:265-9.

9. Katayama T, Shimizu J, Suda K, Onozato R, Fukui T, Ito S, et al. Efficacy of erlotinib for brain and leptomeningeal metastases in patients with lung adenocarcinoma who showed initial good response to gefitinib. J Thorac Oncol 2009;4:1415-9.

10. Lee KY, Kim HJ, Kim SJ, Yoo GH, Kim WD, Oh SY, et al. PNA-mediated PCR clamping for the detection of EGFR mutations in non-small cell lung cancer. Tuberc Respir Dis 2010;69:271-8. Korean.

11. Kim HJ, Oh SY, Kim WS, Kim SJ, Yoo GH, Kim WD, et al. Clinical investigation of EGFR mutation detection by pyrosequencing in lung cancer patients. Oncol Lett 2013;5:271-6.

12. Shingyoji M, Kageyama H, Sakaida T, Nakajima T, Matsui Y, Itakura $\mathrm{M}$, et al. Detection of epithelial growth factor receptor mutations in cerebrospinal fluid from patients with lung adenocarcinoma suspected of neoplastic meningitis. J Thorac Oncol 2011;6:1215-20.

13. Kim HJ, Lee KY, Kim YC, Kim KS, Lee SY, Jang TW, et al. Detection and comparison of peptide nucleic acid-mediated real-time polymerase chain reaction clamping and direct gene sequencing for epidermal growth factor receptor mutations in patients with non-small cell lung cancer. Lung Cancer 2012;75:321-5.

14. Kim HR, Lee SY, Hyun DS, Lee MK, Lee HK, Choi CM, et al. Detection of EGFR mutations in circulating free DNA by PNA-mediated PCR clamping. J Exp Clin Cancer Res 2013; 32:50.

15. Aisner DL, Sams SB. The role of cytology specimens in molecular testing of solid tumors: techniques, limitations, and opportunities. Diagn Cytopathol 2012;40:511-24. 